\title{
Local Application of Alendronate on $\beta$-Tricalcium Phosphate Accelerated Induction of Osteogenesis with Formation of Giant Osteoclast-Like Cell
}

\author{
Chisako Fukuda, Norihiro Akiyama, Mitsuru Takemoto, Shunsuke Fujibayashi, Masashi Neo, \\ Takashhi Nakamura
}

Department of Orthopaedic Surgery, Faculty of Medicine, Kyoto University, Kyoto, Japan.

Email: mediswim@gmail.com

Received January $17^{\text {th }}, 2012$; revised February $25^{\text {th }}, 2012$; accepted March $14^{\text {th }}, 2012$

\begin{abstract}
Intrinsic osteoinductivity - the ability to induce bone formation in ectopic sites without addition of osteogenic factorshas been reported in various porous materials. Tartrate-resistant acid phosphatase (TRAP)-positive osteoclast-like cells are thought to play an important role in material-induced osteoinduction. To investigate the influence of osteoclastic activity on intrinsic osteoinduction, we loaded alendronate $\left(10^{-2}, 10^{-4}\right.$, and $\left.10^{-6} \mathrm{M}\right)$ onto porous $\beta$-tricalcium phosphate $(\beta$-TCP) blocks to inhibit osteoclastic activity, and evaluated osteoinductivity by implantation of the blocks into the dorsal muscles of adult beagle dogs. Alendronate-loaded porous $\beta$-TCP blocks increased both speed and amount of osteoinduction, as measured 4 weeks after implantation, with the $10^{-4} \mathrm{M}$ alendronate-loaded $\beta$-TCP being especially active. This finding indicates that $\beta$-TCP loaded with $10^{-4} \mathrm{M}$ alendronate might prove crucial in providing the desirable balance between the degradation rate of bone scaffolds and their osteoinductive replacement. Thus, material-induced osteoinduction may be controlled by local application of alendronate, establishing alendronate loading as a promising therapeutic approach.
\end{abstract}

Keywords: Osteoinduction; $\beta$-tricalcium Phosphate; Alendronate; Osteoclast; Osteogenesis

\section{Introduction}

Various porous materials exhibit intrinsic osteoinductivity, which is the ability to induce bone formation at ectopic sites without addition of osteogenic factors, such as bone morphogenetic proteins (BMPs), or cell transplanttation [1-10]. The osteoinductivity of materials is affected by their composition [11], macro- and micro- pore size [11-15], as well as the animal models employed in its investigation $[4,16,17]$. However, the exact mechanism of intrinsic osteoinduction by bioactive materials is yet to be elucidated.

Porous $\beta$-tricalcium phosphate ( $\beta$-TCP) with interconnected micro-pores is an osteoinductive material that exhibits intrinsic osteoinductivity in canine dorsal muscles [18]. We previously reported that $\beta$-TCP-induced bone formation at ectopic sites was accelerated by injection of an EP4 agonist (prostaglandin E receptor 4, a prostaglandin E2 receptor subtype). Further, the EP4 agonist enhanced the degradation of porous $\beta$-TCP constructs and stimulated osteoclastogenesis before bone formation [19]. Subsequently, in concert with Kondo et al. [18], we considered the crucial role of tartrate-resistant acid phosphatase (TRAP)-positive osteoclast-like cells in material-induced osteoinduction. Enhanced osteoclastic activity results in an increase of $\beta$-TCP replacement by bone. In addition, we reported that TRAP-positive cells might be an important factor responsible for the crossspecies differences in material-induced osteoinduction [17]. While TRAP-positive multinuclear cells were detected and osteoinduction was observed in the dorsal muscles of dogs 4 weeks after the implantation of porous calcium-deficient hydroxyapatite (CDHA), no osteoinduction and only a small number of TRAP-positive cells were observed 6 weeks following the same treatment in rats. Moreover, osteoclast expression of cathepsin $\mathrm{K}$, a cysteine protease that degrades type-I collagen osteoid matrix, was higher in dogs than in rats.

In the present study, to further investigate the influence of osteoclastic activity on intrinsic osteoinduction, we loaded alendronate onto porous $\beta$-TCP blocks. We hypothesized that inhibition of osteoclastic activity by alendronate results in a decrease of $\beta$-TCP replacement by forming bone tissue. Alendronate, a nitrogencontaining bisphosphonate, is one of the most common thera- 
peutic agents used in the treatment of osteoporosis caused by osteoclastic bone resorption. This bisphosphonate suppresses osteoclastic activity by inhibiting protein isoprenylation through inhibition of geranylgeranylation and, to some extent, farnesylation [20]. Osteoclast precursors treated with alendronate exhibited diminished differenttiation, disruption in their cytoskeleton, and higher level of apoptosis, all associated with inhibition of geranylgeranylation, ultimately leading to suppression of bone resorption [21]. While the clinical efficacy of alendronate is widely believed to result from its potent ability to decrease the number of osteoclasts by promoting their apoptosis, its mechanism of action in vivo may prove different altogether because the number of osteoclasts in cancellous bone specimens obtained from patients treated with alendronate changed little [22] or increased rather than decreased [23]. Thus, the mechanism by which alendronate works in vivo remains controversial. Additionally, several studies dealing with the effect of bisphosphonates on osteoconduction by materials have been conducted. Tanaka et al. reported that when $\beta$-TCP treated with alendronate was implanted in the distal femur of rabbits, the number of osteoclasts on the material decreased and the osteoconduction was inhibited in a dosedependent manner [24]. On the other hand, Bobyn et al. reported that intravenous injection of zoledronic acid enhanced bone growth into porous tantalum materials implanted into the intramedullary canal of the ulna of dogs [25]. Thus, the effect of bisphosphonates on osteoconduction by materials also varies, although these results cannot simply be compared to each other, because of many differences in materials, animal species, bisphosphonate drugs, and treatment methods between the reports.

This study investigated the influence of osteoclastic activity on intrinsic osteoinduction and assessed whether local application of alendronate on porous $\beta$-TCP blocks reduces the amount and activity of TRAP-positive osteoclast-like cells, and consequently inhibits material-induced osteoinduction in ectopic sites.

\section{Materials and Methods}

\subsection{Materials}

\subsubsection{Preparation of Microporous/Macroporous $\beta$-TCP Blocks}

Porous $\beta$-TCP blocks (OSferion ${ }^{\circledR}$ cylinders; porosity, $75 \%$; average pore size, $200 \mu \mathrm{m}$; $\varnothing, 4 \mathrm{~mm}$; height, $8 \mathrm{~mm}$ ) were supplied by Olympus Terumo Biomaterials Corp. (Tokyo, Japan) (Figure 1(a)). These porous $\beta$-TCP blocks were manufactured as follows: $\beta$-TCP powder was synthesized mechanochemically by wet milling. $\mathrm{CaHPO}_{4}$ $\mathrm{H}_{2} \mathrm{O}$ and $\mathrm{CaCO}_{3}$ at a molar ratio of 2:1 were mixed into a slurry with pure water and particles of zirconium in a pot mill for $24 \mathrm{~h}$, and dried at $80^{\circ} \mathrm{C}$, leading to formation of calcium-deficient hydroxyapatite. This crystalline solid was converted to $\beta$-TCP by calcination at $750^{\circ} \mathrm{C}$ for $1 \mathrm{~h}$. After sintering of $\beta$-TCP powder at $1050^{\circ} \mathrm{C}$ for $1 \mathrm{~h}$, a porous $\beta$-TCP block was obtained. The porosity of the block was $75 \%$ and the surface area was $1.4 \mathrm{~m}^{2} / \mathrm{g}$. The morphology of microporous/macroporous $\beta$-TCP block was examined by scanning electron microscopy (SEM) (S-4700, Hitachi Ltd., Tokyo, Japan). The $\beta$-TCP block possessed macropores $(100-500 \mu \mathrm{m})$ that were interconnected via $100-200 \mu \mathrm{m}$ pores, and micropores $(<5 \mu \mathrm{m})$ (Figure 1(b)).

\subsubsection{Preparation of Alendronate-Loaded Porous $\beta$-TCP Blocks}

Alendronate sodium hydrate $\left(\right.$ Teiroc $^{\circledR}$ Injection) was supplied by Teijin Pharma Ltd. (Tokyo, Japan). Thirtysix porous $\beta$-TCP blocks were used. For histological evaluation, $6 \beta$-TCP blocks were immersed in undiluted alendronate solution $\left(10^{-2} \mathrm{M}\right)$, alendronate solutions diluted with saline $\left(10^{-4}\right.$ and $\left.10^{-6} \mathrm{M}\right)$, and pure saline (control). Additionally, for quantitative real-time polymerase chain reaction (qRT-PCR) analysis of osteoclastic gene expression, we compared the $\beta$-TCP blocks pre-loaded with the most dense, undiluted alendronate solution with the control. Each group of $6 \beta$-TCP blocks was immersed in undiluted alendronate solution $\left(10^{-2} \mathrm{M}\right)$ and saline (control), respectively. Each $\beta$-TCP block was immersed in $1.5 \mathrm{~mL}$ of the corresponding solution. Solutions containing $\beta$-TCP blocks were then vacuumized with an oil rotary vacuum pump (G-25SA; ULVAC KIKO Inc., Miyazaki, Japan) for 15 minutes. After vacuumization, the $\beta$-TCP blocks were kept immersed in the solutions for 2 days at $4^{\circ} \mathrm{C}$. Subsequently, the blocks were removed from solutions to dry at room temperature.

\subsubsection{Loading Alendronate on and Release of Alendronate from Porous $\beta$-TCP Blocks}

Radioactive samples were prepared according to the aforementioned procedure by using ${ }^{14} \mathrm{C}$-labeled alendro-
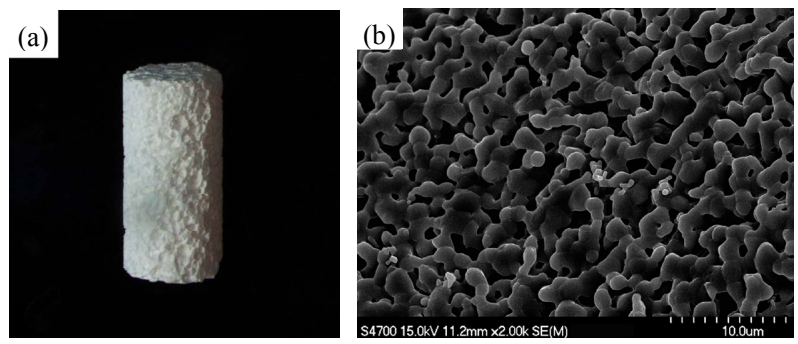

Figure 1. (a) Porous $\beta$-TCP cylinder (diameter $=4 \mathrm{~mm}$, length $=8 \mathrm{~mm}$ ); (b) Porous $\beta$-TCP cylinder possesses micropores of less than $5 \mu \mathrm{m}$. 
nate (Moravek Biochemicals Inc., California, USA). Six $\beta$-TCP blocks were immersed in solutions of ${ }^{14} \mathrm{C}$-labeled alendronate. The loading efficiency of alendronate on $\beta$-TCP blocks was calculated as the difference between its initial concentration and its residual concentration in the supernatant. In order to determine the amount of alendronate released from alendronate-loaded $\beta$-TCP, ${ }^{14} \mathrm{C}$ alendronate-loaded $\beta$-TCP blocks were immersed in 4 $\mathrm{mL}$ of culture medium, and the alendronate concentration was evaluated by liquid scintillation after 1,4 , and 7 days. At the predetermined time intervals, the samples (1 $\mathrm{mL}$ ) were collected and replaced with equal volumes of fresh medium.

\subsection{Canine Model for Osteoinduction}

Osteoinductivity was evaluated by implantation of porous $\beta$-TCP blocks into the dorsal muscles of adult beagle dogs. Four healthy, 1-year-old, female beagle dogs weighing $10 \mathrm{~kg}$ were used for the study. The animals were reared and the experiments were carried out at the Institute of Laboratory Animals, Graduate School of Medicine, Kyoto University, Japan. All procedures were performed according to the Principles of Laboratory Animal Care of the Kyoto University Animal Experiment Committee. The dogs were anesthetized by intramuscular administration of ketamine hydrochloride $(7.5 \mathrm{mg} / \mathrm{kg})$ and xylazine $(7 \mathrm{mg} / \mathrm{kg})$. The operations were performed under standard sterile conditions. After performing a longitudinal incision in the skin and fascia in the middle of the back, intramuscular pouches were created in the bilateral dorsal muscles. For histological evaluation, 8 $\beta$-TCP blocks pre-loaded with alendronate $\left(10^{-2}, 10^{-4}\right.$, or $10^{-6} \mathrm{M}$ ) or saline (control) were implanted in 8 intramuscular pouches of 3 dogs. For qRT-PCR analysis, 12 $\beta$-TCP blocks pre-loaded with alendronate $\left(10^{-2} \mathrm{M}\right)$ or saline were implanted in 12 intramuscular pouches of the fourth dog. The fascia and skin were sutured in layers after irrigation with saline. The $\beta$-TCP samples were completely covered with muscle and dispersed sufficiently not to affect each other.

\subsection{Histological Examinations and Histomorphometry}

For histological evaluation, appropriate animals were anesthetized and the implants were retrieved after 4 weeks. The samples were fixed in $4 \%$ neutral buffered paraformaldehyde (PFA) for $48 \mathrm{~h}$, decalcified with a $20 \%$ EDTA solution at $4{ }^{\circ} \mathrm{C}$ for approximately a week, dehydrated in an ethanol series $(70 \%, 80 \%, 90 \%, 99.5 \%$, and $100 \%$ ), and embedded in paraffin. The samples were sectioned perpendicular to the long axis of the cylinder by using a microtome (SM2000R; Leica Ltd., Tokyo,
Japan) to yield 4 pairs of adjacent 5 - $\mu \mathrm{m}$-thick sections. The first pair of sections was approximately 1-mm apart from the end of the cylinder sample, and each subsequent pair of sections was approximately 1-mm apart from the previous one. Half of the section-pairs were stained with hematoxylin and eosin (HE), and the remaining sections were stained with TRAP staining kit (MK300; Takara Bio Inc., Shiga, Japan) to detect osteoclast-like multinuclear cells. Images were acquired with Nikon Eclipse 80i (Tokyo, Japan) light microscope and analyzed using Adobe Photoshop CS5 (Adobe Systems Inc., CA, USA) and ImageJ (National Institutes of Health freeware). The area of newly formed bone, residual $\beta$-TCP, and the porous area of $\beta$-TCP were calculated in each section stained with HE, and multinuclear TRAP-positive cells (with more than 3 nuclei) were counted in each section stained with TRAP. New bone formation rate (\%) was calculated by dividing the area of newly formed bone by the porous area of $\beta$-TCP and multiplying this value by 100 .

\subsection{Relative Quantitative Real-time Polymerase Chain Reaction (qRT-PCR) Analyses}

To correlate the morphometric evaluation of materialinduced osteoinduction with osteoclastic gene expression, the appropriate samples were analyzed for changes in TRAP and cathepsin K mRNA synthesis by qRT-PCR. The $12 \beta$-TCP implants, harvested at 2,4 , and 8 weeks (and immediately frozen in liquid nitrogen), were crushed in a mortar filled with liquid nitrogen. Total RNA was isolated using TRIzol ${ }^{\circledR}$ Reagent (Invitrogen Co., CA, USA) and further purified using RNeasy $^{\circledR}$ Mini kit (Qiagen Inc., CA, USA) according to the manufacturer's instructions. First-strand cDNA was prepared from $1 \mu \mathrm{g}$ of total RNA by using Transcriptor First Strand cDNA Synthesis Kit (Roche Diagnostics, Mannheim, Germany), and qRT-PCR was performed using LightCycler ${ }^{\circledR} 1.5$ with LightCycler ${ }^{\circledR}$ FastStart DNA Master ${ }^{\text {PLUS }}$ SYBR Green I (Roche Diagnostics) according to the manufacturer's instructions. The sequences of primers used for the amplification of the housekeeping glyceraldehyde 3-phosphate dehydrogenase (GAPDH) gene, as well as the osteoclast marker genes encoding cathepsin $\mathrm{K}$ and TRAP, are shown in Table 1. Quantitative data analyses were performed with the LightCycler software 3.5 (Roche Diagnostics). Relative expression levels of TRAP and cathepsin K genes were normalized to GAPDH.

\subsection{Statistical Analyses}

All values are expressed as mean \pm standard deviation. The data concerning new bone formation rate, residual amount of $\beta$-TCP, and amount of TRAP-positive mul- 
tinuclear cells were analyzed using the Steel-Dwass procedure with a 1-way design for nonparametric all-pairs multiple comparisons. The qRT-PCR data were analyzed using the Mann-Whitney's $U$ test. The critical level of statistical significance was $\mathrm{p}<0.05$.

\section{Results}

\subsection{Amount of Alendronate Loaded on and Released from Porous $\beta$-TCP Blocks}

The amount of alendronate loaded on and released from the porous $\beta$-TCP blocks was evaluated using ${ }^{14} \mathrm{C}$-labeled alendronate. The results indicated that $67.4 \% \pm 1.6 \%$ of the initial amount of alendronate solution was incorporated on porous $\beta$-TCP blocks. After 1-, 4-, and 7-day immersion, cumulative alendronate release (\%) into the culture medium was $0.21 \pm 0.07,0.39 \pm 0.12$, and $0.79 \pm$ 0.34, respectively (Figure 2).

\subsection{Induction of Bone Formation by Alendronate-Loaded $\beta$-TCP Blocks}

After 4 weeks, 3 of the 6 implants from the $10^{-2} \mathrm{M}$ group, 4 of the 6 implants from the $10^{-4} \mathrm{M}$ group, and 2 of the 6 implants from the $10^{-6} \mathrm{M}$ group showed osteoinduction, while the process was not observed in the control group (Table 2). Induction of bone formation was more remarkable in the peripheral zone of porous $\beta$-TCP than in the center (Figure 3(a)). Newly formed bone tissue appeared to spread not only along the surface of $\beta$-TCP, but also within the macroporous spaces, where active angiogenesis was identified (Figure 3(b)). Moreover, cuboidal osteoblast-like cells were lining along induced bones. Osteoclast-like multinuclear cells were also identified on the $\beta$-TCP surface in HE stained sections. Again, the formation of osteoblasts and osteoclasts, as well as cap- illaries, was more abundant in the peripheral zone than in the central zone. In porous spaces of $\beta$-TCP, collagenous condensations of extracellular matrix were observed (Figure 3(c)). New bone formation rate (\%) in the $10^{-4} \mathrm{M}$ group was significantly higher than those in the $10^{-2} \mathrm{M}$ and $10^{-6} \mathrm{M}$ groups (Figure 4).

Table 1. The sequences of the primers.

\begin{tabular}{ll}
\hline Gene & Sequence $\left(5^{\prime}-3^{\prime}\right)$ \\
\hline \multirow{2}{*}{ GAPDH } & F: AACATCATCCCTGCTTCCAC \\
& R: GACCACCTGGTCCTCAGTGT \\
Cathepsin K & F: AAGAACCAGGGTCAGTGTGG \\
& R: GAGGTCAGGCTTGCATCAAT \\
TRAP & F: GATGCCAACGACAAGAGGTT \\
& R: CTTTGAAGCGTAGCCGGTAG \\
\hline
\end{tabular}

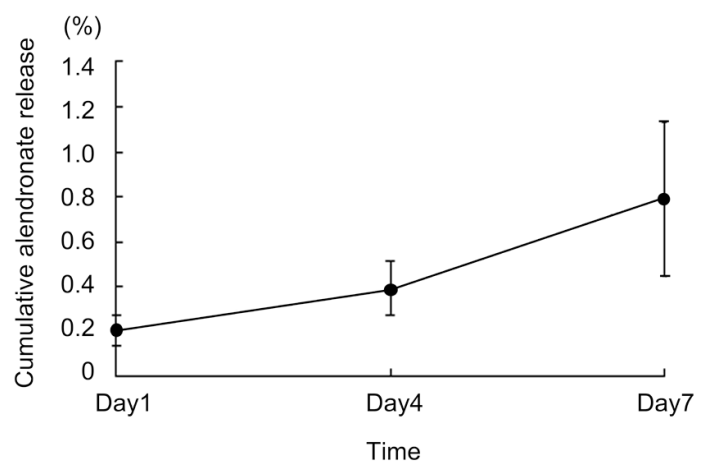

Figure 2. Cumulative alendronate release (\%) of alendronate-loaded porous $\beta$-TCP blocks in the culture medium.

Table 2. Number of samples that showed induction of bone formation.

\begin{tabular}{lllll}
\hline Group & $10^{-2} \mathrm{M}$ & $10^{-4} \mathrm{M}$ & $10^{-6} \mathrm{M}$ & Control \\
\hline Number of samples & $3 / 6$ & $4 / 6$ & $2 / 6$ & $0 / 6$ \\
\hline
\end{tabular}
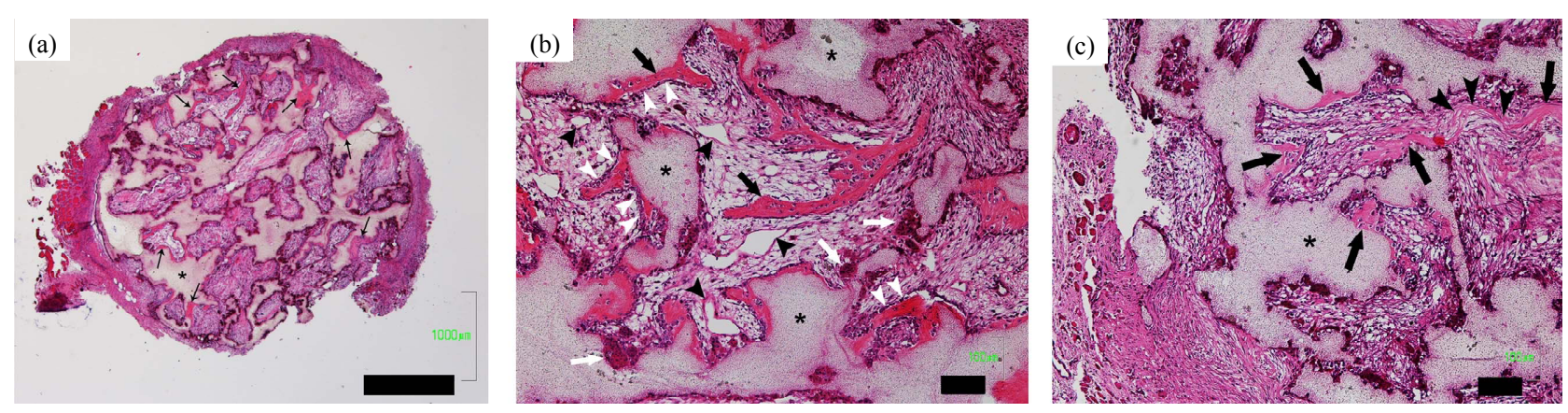

Figure 3. Osteoinduction of porous $\beta$-TCP blocks loaded with alendronate $\left(10^{-4} \mathrm{M}\right) 4$ weeks after implantation. (a) Induction of bone (black arrows) was found in a peripheral area of materials more than a central area. Bar $=1000 \mu \mathrm{m} .{ }^{*}, \beta$-TCP; $(\mathrm{b})$ High magnification image of (a) showed that osteoinduction (black arrows) extended along the surface of $\beta$-TCP (*) and also within the macroporous spaces where angiogenesis (black arrow heads) was active. Osteoclast-like multinuclear cells (white arrows) attached to a surface of $\beta$-TCP. Osteoblast-like cells (white arrow heads) were lining along newly formed bone. Bar $=$ $100 \mu \mathrm{m}$; (c) High magnification image of another specimen showed induced bones (black arrows) and collagenous condensations of extracellular matrix (black arrow heads). Bar $=100 \mu \mathrm{m}$. *, $\beta$-TCP. 


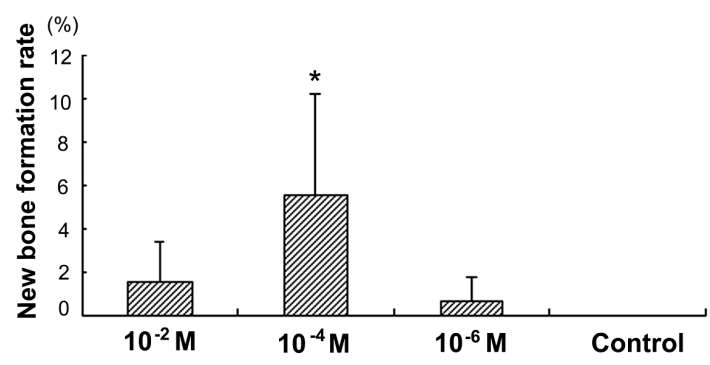

Figure 4. New bone formation rate $(\%)$ of porous $\beta$-TCP blocks loaded with alendronate $\left(10^{-2} \mathrm{M}, 10^{-4} \mathrm{M}\right.$ and $\left.10^{-6} \mathrm{M}\right)$ and saline (control) 4 weeks after implantation. There was no new bone formation at the control group. *Significant compared with the $10^{-2} \mathrm{M}$ and $10^{-6} \mathrm{M}$ groups $(\mathrm{p}<0.05)$.

\subsection{Residual Amount of Porous $\beta$-TCP}

After 4 weeks, the residual $\beta$-TCP area in the control group was the smallest and significantly different from those in the alendronate-loaded groups (Figure 5). The residual $\beta$-TCP area in the alendronate-loaded groups increased with the density of alendronate; however, there were no significant differences between the alendronateloaded groups (Figure 5).

\subsection{Histomorphometric Features and Amount of TRAP-Positive Multinuclear Cells}

In the alendronate-loaded groups, giant TRAP-positive osteoclast-like cells with more than 10 nuclei were found (Figures 6(a) and (b)). In addition, giant osteoclast-like cells often detached from $\beta$-TCP surface, and other mononuclear cells, interposed between the $\beta$-TCP surface and the giant osteoclast-like cells, were observed; this finding proves that the detachment was not due to tissue shrinkage during histologic preparation (Figure 6(a)). On the other hand, no giant osteoclast-like cells were found, and the TRAP-positive multinuclear cells were

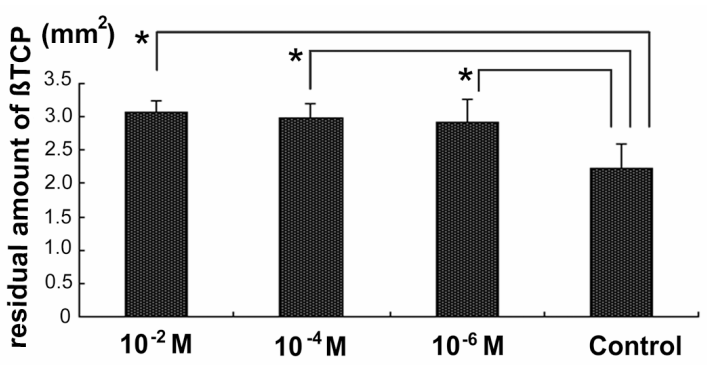

Figure 5. Residual amount of porous $\beta$-TCP blocks with alendronate $\left(10^{-2} \mathrm{M}, 10^{-4} \mathrm{M}\right.$ and $\left.10^{-6} \mathrm{M}\right)$ and saline (control) 4 weeks after implantation. *Significant compared with the control group $(p<0.05)$.

always tightly adherent to the $\beta$-TCP surface in the control group (Figure 6(c)). After 4 weeks, the amount of TRAP-positive multinuclear cells divided by the remaining amount of $\beta$-TCP in the alendronate-loaded groups was smaller than in the control; however, no significant differences between the groups were observed (Figure 7). Among the alendronate-loaded groups, the amount of TRAP-positive multinuclear cells was lowest in the $10^{-2}$ $\mathrm{M}$ group. However, the obvious tendency to decrease the number of TRAP-positive multinuclear cells by increasing the amount of loaded alendronate was not seen.

\subsection{Relative Quantitative Real-Time Polymerase Chain Reaction (qRT-PCR) Analyses}

qRT-PCR analysis showed that the expression of TRAP was decreased in the $\beta$-TCP specimens loaded with alendronate (Figure 8). Expression of cathepsin K was also slightly decreased in the alendronate-loaded specimens. There were no significant differences between the control and the $10^{-2} \mathrm{M}$ group in the same harvest period. In both groups, the expression of TRAP and cathepsin K increased with time, and the expression of TRAP was
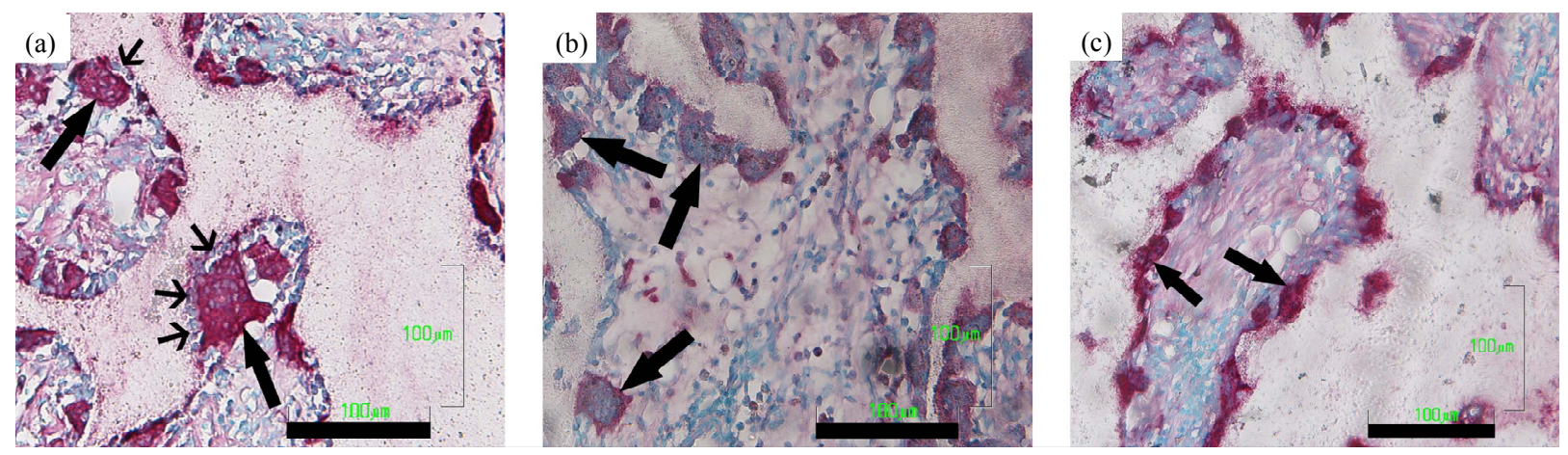

Figure 6. TRAP staining sections at 4 weeks. (a) The $10^{-2} \mathrm{M}$ group; giant TRAP-positive multi-nuclear cells with more than 10 nuclei (black wide arrows) were found. Some of them detached from the $\beta$-TCP surface and there were mononuclear cells interposed between the $\beta$-TCP surface and the giant osteoclast-like cell (black narrow arrows). Bar $=100 \mu \mathrm{m} ;(\mathrm{b}) \mathrm{The} 10^{-4} \mathrm{M}$ group; Giant and hypernucleated TRAP-positive multi-nuclear cells were found (black arrows). Bar $=100 \mu \mathrm{m}$; (c) The control group; TRAP-positive multi-nuclear cells were tightly adherent to the $\beta$-TCP surface (black arrows). Bar $=100 \mu \mathrm{m}$. 


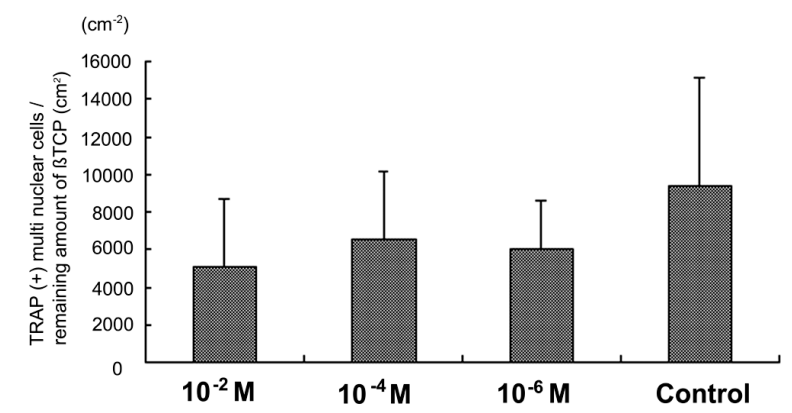

Figure 7. The amount of TRAP-positive multi nuclear cells revised by remaining amount of $\beta$-TCP in porous $\beta$-TCP blocks pre-loaded with alendronate $\left(10^{-2} \mathrm{M}, 10^{-4} \mathrm{M}\right.$ and $\left.10^{-6} \mathrm{M}\right)$ and saline (control) harvested at 4 weeks.

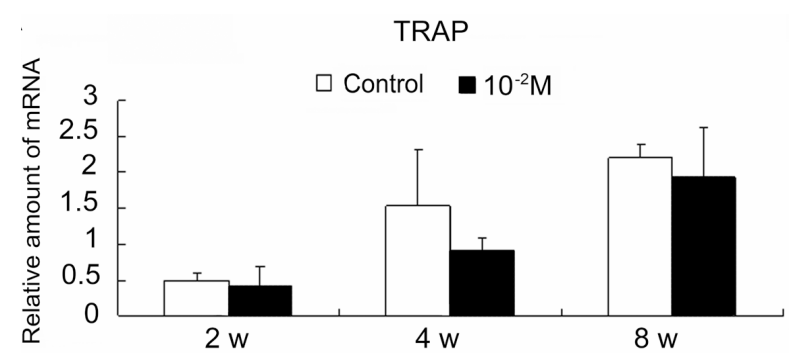

(a)

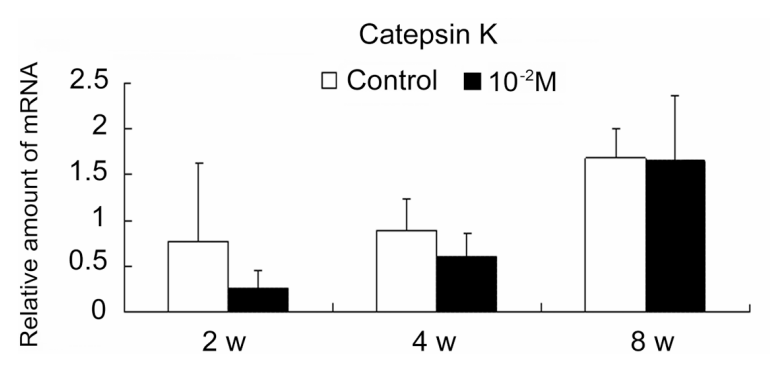

(b)

Figure 8. Effect of alendronate on osteoclastic gene expression using quantitative real-time polymerase chain reaction (qRT-PCR). TRAP (a) and Catepsin K (b) mRNA expression in the control group and the alendronate $\left(10^{-2} \mathrm{M}\right)$ loaded group retrieved at 2, 4 and 8 weeks after implantation.

greater than that of cathepsin $\mathrm{K}$, except in the control group after 2 weeks.

\section{Discussion}

Although we hypothesized that osteoinduction by alendronate-loaded materials decreases because of the inhibited osteoclastic activity, our study disclosed that local application of alendronate enhances material-induced osteoinduction by porous $\beta$-TCP. Bone formation induced by porous $\beta$-TCP without any additional osteogenic factors was usually observed 6-8 weeks after implantation in heterotrophic sites $[18,19]$. However, as reported in the present study, porous $\beta$-TCP blocks pre-loaded with alendro- nate $\left(10^{-2} \mathrm{M}, 10^{-4} \mathrm{M}\right.$, and $\left.10^{-6} \mathrm{M}\right)$ induced osteogenesis as early as 4 weeks after implantation in dorsal muscles of canines, while no osteoinduction was observed at that time-point in the control group.

Ripamonti et al. [26] reported that local application of zoledronate onto coral-derived hydroxyapatite/calcium carbonate macroporous constructs $(7 \% \mathrm{HA} / \mathrm{CC})$ inhibited osteoinduction 2 months after implantation in heterotopic sites of Papio ursinus. Conversely, the results of this study indicated that local application of alendronate on $\beta$ TCP blocks enhanced osteoinduction after 4 weeks much earlier than in the aforementioned report. We contend that this difference in effect of bisphosphonate on osteoinduction depends mainly on the material characteristics. Ripamonti et al. used macroporous materials without microporous structures, and indicated that osteoinduction was inhibited by zoledronate as the osteoclastic post-implantation modifications of the materials decreased, which was followed by the reduction of the macro- and micro- patterned topographies, important for the differentiation of resident stem cells into osteoblastlike cells. We, however, used $\beta$-TCP blocks possessing microporous structures on the macropore walls. Consequently, this original topography proved essential for increased osteoinduction observed in this study.

Induction of bone formation was more remarkable in the peripheral zone of the porous $\beta$-TCP, where the formation of osteoblasts, osteoclasts, and capillaries was more abundant than in the center. In the macroporous area, mesenchymal collagenous condensations of extracellular matrix were also observed. They are considered the source of tissue patterning and morphogenesis, ultimately promoting osteogenesis within the macroporous materials [27]. These findings suggest that the capillaries generated in the peripheral zone of the porous $\beta$-TCP contribute to bone induction through advancement of osteoblast and osteoclast formation and tissue morphogenesis. In addition, we observed newly formed bone tissue spread from pore surface to the central part of macroporous spaces in the alendronate groups, as shown in Figure 3(b). While different from the results obtained by Kondo et al. [18], who indicated that a newly forming bone induced by a single $\beta$-TCP implant was always directly attached to the material surface, our finding is consistent with that of Nasu's report [19], which suggests enhanced osteoinduction by EP4 agonist spread within macroporous spaces of $\beta$-TCP. Cumulatively, all aforementioned data indicate that osteoinduction by $\beta$-TCP initially occurs on the pore surface and subsequently extends to the central zone of porous spaces, as the process accelerates.

We found that the amount of alendronate released from $\beta$-TCP blocks was low. This result indicates that 
most of the loaded alendronate was combined with the blocks after implantation, and that alendronate probably acts on osteoclast-like cells attached to the surface of $\beta$-TCP. Moreover, giant, hypernucleated, and detached osteoclast-like cells were found in the sections of the alendronate-loaded implants. The histomorphometric features of osteoclast-like cells reported in this study are the same as those found in the study of bone-biopsy specimens obtained from patients treated with orally administered alendronate, in which the giant, hypernucleated, and detached osteoclasts were observed to undergo protracted apoptosis [23]. Osteoclasts are produced by the fusion of mononuclear precursors and usually acquire up to 8 nuclei before dying by apoptosis, probably as a result of exposure to the high extracellular concentration of released calcium occurring during bone resorption [28, 29]. Inhibition of $\beta$-TCP resorption by alendronate would decrease the signal for apoptosis of osteoclast-like cells resulting from calcium release, consequently prolonging the duration of apoptosis and extending the time for fusion of osteoclast-like cells with additional mononuclear progenitors [23].

Duque et al. reported that alendronate exerted an anabolic effect through the stimulation of osteogenic differentiation of human mesenchymal stem cells (MSCs) [30]. What is more, while the inhibitive activity of nitrogencontaining bisphosphonates on osteoblastogenesis using mature calvarial osteoblasts was reported [31,32], a more recent report has shown that alendronate potentiated the effect of geranylgeranyltransferase inhibitor on geranylgeranylation, as well as reverted the effect of farnesyltransferase inhibitor on protein farnesylation, both in favor of osteoblastogenesis [33]. In light of the stimulating effect of alendronate on osteogenic differentiation of MSCs, loading $\beta$-TCP blocks with alendronate may contribute to the enhancement of ectopic osteogenesis in the porous $\beta$-TCP through activation of the MSCs in muscles around implants.

A particularly interesting finding of this study indicated that pre-loading the porous $\beta$-TCP blocks with $10^{-4}$ $\mathrm{M}$ alendronate solution most significantly enhanced osteoinduction. We believe that the $10^{-4} \mathrm{M}$ alendronateloaded $\beta$-TCP proved crucial in providing the desirable balance between the degradation rate of bone scaffolds and their osteoinductive replacement, under the conditions of our study. Whereas high biodegradability and solubility are characteristics important for $\beta$-TCP to induce angiogenesis [34], osteoblastogenesis [35], and bone formation, the prolonged exposure to high concentration of $\mathrm{Ca}^{2+}$ and phosphate ions released from $\beta$-TCP is detrimental to cells, including osteoblasts, osteoclasts, and osteocytes, in vivo [36]. Therefore, the concentration of $\mathrm{Ca}^{2+}$ and phosphate ions released from the $10^{-4} \mathrm{M}$ al- endronate-loaded blocks seems to be advantageous for both induction of osteogenesis and cell survival.

Finally, the role of osteoclastic activity in materialinduced osteoinduction in vivo, as well as the entire mechanism of material-induced osteoinduction remains to be elucidated. Lack of significant suppression of osteoclastic activity after local application of alendronate, even at its highest undiluted density $\left(10^{-2} \mathrm{M}\right)$, as indicated by qRT-PCR analysis results (Figure 8), constitutes a serious limitation of our study. Significant inhibittion of osteoclastogenesis at an early stage, e.g., by means of RANKL (receptor activator of nuclear factor $\kappa$-B ligand) antibodies, could prove an interesting approach to further investigation of the influence of osteoclastic activity on intrinsic osteoinduction in vivo.

\section{Conclusion}

This study showed that loading alendronate on porous $\beta$ TCP blocks increased both speed and amount of material-induced osteoinduction of $\beta$-TCP after implantation in heterotopic dorsal muscles of canines. The $10^{-4} \mathrm{M}$ alendronate-loaded $\beta$-TCP displayed the highest activity, inducing a significantly large amount of new bone formation. We contend that $\beta$-TCP loaded with $10^{-4} \mathrm{M}$ alendronate proved crucial in providing the desirable balance between the degradation rate of bone scaffolds and their osteoinductive replacement in this study. Thus, ectopic material-induced osteoinduction may be controlled by local application of alendronate, establishing alendronate loading as a promising therapeutic approach.

\section{Disclosures}

Olympus Terumo Biomaterials Corp. provided porous $\beta$ TCP blocks samples and Teijin Pharma Ltd. provided alendronate. However, there is no conflict of interest related to the work.

\section{Acknowledgements}

We thank Olympus Terumo Biomaterials Corp. and Teijin Pharma Ltd. for supplying $\beta$-TCP and alendronate, respectively. We greatly appreciate the technical support of Haruyasu Kohda and Keiko Furuta for the SEM studies and Hideo Miyatake for the radioisotope studies.

\section{REFERENCES}

[1] G. D. Winter and B. J. Simpson, "Heterotopic Bone Formed in a Synthetic Sponge in the Skin of Young Pigs," Nature, Vol. 223, 1969, pp. 88-90. doi: $10.1038 / 223088 \mathrm{a} 0$

[2] U. Ripamonti, "The Morphogenesis of Bone in Replicas of Porous Hydroxyapatite Obtained from Conversion of 
Calcium Carbonate Exoskeletons of Coral," Journal of Bone and Joint Surgery, Vol. 73, No. 5, 1991, pp. 692703.

[3] H. Yamasaki and H. Sakai, "Osteogenic Response to Porous Hydroxyapatite Ceramics under the Skin of Dogs," Biomaterials, Vol. 13, No. 5, 1992, pp. 308-312. doi:10.1016/0142-9612(92)90054-R

[4] U. Ripamonti, "Osteoinduction in Porous Hydroxyapatite Implanted in Heterotopic Sites of Different Animal Models," Biomaterials, Vol. 17, No. 1, 1996, pp. 31-35. doi:10.1016/0142-9612(96)80752-6

[5] H. Yuan, Z. Yang, Y. Li, X. Zhang, J. D. De Bruijn and K. De Groot, "Osteoinduction by Calcium Phosphate Biomaterials," Journal of Materials Science: Materials in Medicine, Vol. 9, No. 12, 1998, pp. 723-726. doi:10.1023/A:1008950902047

[6] H. Yuan, Y. Li, J. D. de Bruijn, K. de Groot and X. Zhang, "Tissue Responses of Calcium Phosphate Cement: A Study in Dogs," Biomaterials, Vol. 21, No. 12, 2000 pp. 1283-1290. doi:10.1016/S0142-9612(00)00016-8

[7] H. Yuan, J. D. de Bruijn, X. Zhang, C. A. van Blitterswijk and K. de Groot, "Bone Induction by Porous Glass Ceramic Made from Bioglass (45S5)," Journal of Biomedical Materials Research, Vol. 58, No. 3, 2001, pp. 270-276. doi:10.1002/1097-4636(2001)58:3<270::AID-JBM1016> 3.0.CO;2-2

[8] P. Habibovic, C. M. van der Valk, C. A. van Blitterswijk, K. De Groot and G. Meijer, "Influence of Octacalcium Phosphate Coating on Osteoinductive Properties of Biomaterials," Journal of Materials Science: Materials in Medicine, Vol. 15, No. 4, 2004, pp. 373-380. doi:10.1023/B:JMSM.0000021104.42685.9f

[9] D. Le Nihouannen, G. Daculsi, A. Saffarzadeh, O. Gauthier, S. Delplace, P. Pilet, et al., "Ectopic Bone Formation by Microporous Calcium Phosphate Ceramic Particles in Sheep Muscles," Bone, Vol. 36, No. 6, 2005, pp. 10861093. doi:10.1016/j.bone.2005.02.017

[10] S. Fujibayashi, M. Neo, H. M. Kimb, T. Kokubo and T. Nakamura, "Osteoinduction of Porous Bioactive Titanium Metal," Biomaterials, Vol. 25, No. 3, 2004, pp. 443-450. doi:10.1016/S0142-9612(03)00551-9

[11] Z. Zhang, H. Kurita, H. Kobayashi and K. Kurashina, "Osteoinduction with HA/TCP Ceramics of Different Composition and Porous Structure in Rabbits," Oral Science International, Vol. 2, No. 2, 2005, pp. 85-95.

[12] H. Yuan, K. Kurashina, J. D. de Bruijn, Y. Li, K. de Groot and X. Zhang, "A Preliminary Study on Osteoinduction of Two Kinds of Calcium Phosphate Ceramics," Biomaterials, Vol. 20, No. 19, 1999, pp. 1799-1806. doi:10.1016/S0142-9612(99)00075-7

[13] M. C. Kruyt, W. J. Dhert, C. Oner, C. A. van Blitterswijk and A. J. Verbout, J. D. de Bruijn, "Optimization of Bone-Tissue Engineering in Goats," Journal of Biomedical Materials Research Part B: Applied Biomaterials, Vol. 69, 2004, No. 2, pp. 113-120. doi:10.1002/jbm.b.10073

[14] A. Fukuda, M. Takemoto, T. Saito, S. Fujibayashi, M. Neo, D. K. Pattanayak, et al., "Osteoinduction of Porous
Ti Implants with a Channel Structure Fabricated by Selective Laser Melting," Acta Biomaterialia, Vol. 7, No. 5, 2011, pp. 2327-2336. doi:10.1016/j.actbio.2011.01.037

[15] P. Habibovic, H. Yuan, C. M. van der Valk, G. Meijer, C. A. van Blitterswijk and K. de Groot, "3D Microenvironment as Essential Element for Osteoinduction by Biomaterials," Biomaterials, Vol. 26, No. 17, 2005, pp. 3565-3575. doi:10.1016/j.biomaterials.2004.09.056

[16] Z. Yang, H. Yuan, W. Tong, P. Zou, W. Chen and X. Zhang, "Osteogenesis in Extraskeletally Implanted Porous Calcium Phosphate Ceramics: Variability among Different Kinds of Animals," Biomaterials, Vol. 17, No. 22, 1996, pp. 2131-2137. doi:org/10.1016/0142-9612(96)00044-0

[17] N. Akiyama, M. Takemoto, S. Fujibayashi, M. Neo, M. Hirano and T. Nakamura, "Difference between Dogs and Rats with Regard to Osteoclast-Like Cells in CalciumDeficient Hydroxyapatite-Induced Osteoinduction," Journal of Biomedical Materials Research Part A, Vol. 96, No. 2, 2011, pp. 402-412. doi:org/10.1002/jbm.a.32995

[18] N. Kondo, A. Ogose, K. Tokunaga, H. Umezu, K. Arai, N. Kudo, et al., "Osteoinduction with Highly Purified $\beta$ Tricalcium Phosphate in dog Dorsal Muscles and the Proliferation of Osteoclasts before Heterotopic Bone Formation," Biomaterials, Vol. 27, No. 25, 2006, pp. 44194427. doi:10.1016/j.biomaterials.2006.04.016

[19] T. Nasu, M. Takemoto, N. Akiyama, S. Fujibayashi, M. Neo and T. Nakamura, "EP4 Agonist Accelerates Osteoinduction and Degradation of Beta-Tricalcium Phosphate by Stimulating Osteoclastogenesis," Journal of Biomedical Materials Research Part A, Vol. 89, No. 3, 2009, pp. 601608.

[20] F. P. Coxon, M. H. Helfrich, R. Van't Hof, S. Sebti, S. H. Ralston, A. Hamilton, et al., "Protein Geranylgeranylation is Required for Osteoclast Formation, Function, and Survival: Inhibition by Bisphosphonates and GGTI-298," Journal of Bone and Mineral Research, Vol. 15, No. 8, 2000, pp. 1467-1476. doi:10.1359/jbmr.2000.15.8.1467

[21] R. G. Russell, "Bisphosphonates: From Bench to Bedside," Annals of the New York Academy of Sciences, Vol. 1068, 2006, pp. 367-401. doi:10.1196/annals.1346.041

[22] R. Balena, B. C. Toolan, M. Shea, A. Markatos, E. R Myers, S. C. Lee, et al., "The Effects of 2-Year Treatment with the Aminobisphosphonate Alendronate on Bone Metabolism, Bone Histomorphometry, and Bone Strength in Ovariectomized Nonhuman Primates," Journal of Clinical Investigation, Vol. 92, No. 6, 1993, pp. 25772586. doi:10.1172/JCI116872

[23] R. S. Weinstein, P. K. Roberson and S. C. Manolagas, "Giant Osteoclast Formation and Long-Term Oral Bisphosphonate Therapy," New England Journal of Medicine, Vol. 360, 2009, pp. 53-62. doi:10.1056/NEJMoa0802633

[24] T. Tanaka, M. Saito, M. Chazono, Y. Kumagae, T. Kikuchi, S. Kitasato and K. Marumo, "Effects of Alendronate on Bone Formation and Osteoclastic Resorption after Implantation of Beta-Tricalcium Phosphate," Journal of Biomedical Materials Research Part A, Vol. 93, 
No. 2, 2010, pp. 469-474. doi:10.1002/jbm.a.32560

[25] J. D. Bobyn, S. A. Hacking, J. J. Krygier, E. J. Harvey, D. G. Little and M. Tanzer, "Zoledronic Acid Causes Enhancement of Bone Growth into Porous Implants," Journal of Bone and Joint Surgery British, Vol. 87, No. 3, 2005, pp. 416-420.

[26] U. Ripamonti, R. M. Klar, L. F. Renton and C. Ferretti, "Synergistic Induction of Bone Formation by hOP-1, hTGF- $\beta_{3}$ and Inhibition by Zoledronate in Macroporous Coral-Derived Hydroxyapatites," Biomaterials, Vol. 31, No. 25, 2010, pp. 6400-6410. doi:10.1016/j.biomaterials.2010.04.037

[27] N. Gjorevski and C. M. Nelson, "Bidirectional Extracellular Matrix Signaling during Tissue Morphogenesis," Cytokine \& Growth Factor Reviews, Vol. 20, No. 5-6, 2009, pp. 459-465. doi:10.1016/j.cytogfr.2009.10.013

[28] F. Lorget, S. Kamel, R. Mentaverri, A. Wattel, M. Naassila, M. Maamer, et al., "High Extracellular Calcium Concentrations Directly Stimulate Osteoclast Apoptosis," Biochemical and Biophysical Research Communications, Vol. 268, No. 3, 2000, pp. 899-903. doi:10.1006/bbrc.2000.2229

[29] R. H. Nielsen, M. A. Karsdal, M. G. Sorensen, M. H. Dziegiel and K. Henriksen, "Dissolution of the Inorganic Phase of Bone Leading to Release of Calcium Regulates Osteoclast Survival," Biochemical and Biophysical Research Communications, Vol. 360, No. 4, 2007, pp. 834839. doi:10.1016/j.bbrc.2007.06.145

[30] G. Duque1 and D. Rivas, "Alendronate has an Anabolic Effect on Bone through the Differentiation of Mesenchymal Stem Cells," Journal of Bone and Mineral Research, Vol. 22, No. 10, 2007, pp. 1603-1611.

\section{doi:10.1359/jbmr.070701}

[31] A. I. Idris, J. Rojas, I. R. Greig, R. J. Van't Hof and S. H. Ralston, "Aminobisphosphonates Cause Osteoblast Apoptosis and Inhibit Bone Nodule Formation in Vitro," Calcified Tissue International, Vol. 82, No. 3, 2008, pp. 191201. doi:10.1007/s00223-008-9104-y

[32] I. R. Orriss, M. L. Key, K. W. Colston and T. R. Arnett, "Inhibition of Osteoblast Function in Vitro by Aminobisphosphonates," Journal of Cellular Biochemistry, Vol. 106, No. 1, 2009, pp. 109-118. doi:10.1002/jcb.21983

[33] G. Duque1, C. Vidal and D. Rivas, "Protein Isoprenylation Regulates Osteogenic Differentiation of Mesenchymal Stem Cells: Effect of Alendronate, and Farnesyl and Geranylgeranyl Transferase Inhibitors," British Journal of Pharmacology, Vol. 162, No. 5, 2011, pp. 1109 1118. doi:10.1111/j.1476-5381.2010.01111.X

[34] E. Kohn, R. Alessandro, J. Spoonster, R. P. Wersto and L. A. Liotta, "Angiogenesis: Role of Calcium-Mediated Signal Transduction," Proceedings of the National Academy of Sciences of USA, Vol. 92, No. 5, 1995, pp. 13071311. doi:10.1073/pnas.92.5.1307

[35] M. Zayzafoon, "Calcium/Calmodulin Signaling Controls Osteoblast Growth and Differentiation," Journal of Cellular Biochemistry, Vol. 97, No. 1, 2006, pp. 56-70. doi: $10.1002 / j \mathrm{jcb} .20675$

[36] H. Yuan, J. D. De Bruijn, Y. Li, J. Feng, Z. Yang, K. De Groot, et al., "Bone Formation Induced by Calcium Phosphate Ceramics in Soft Tissue of Dogs: A Comparative Study between Porous $\alpha$-TCP and $\beta$-TCP," Journal of Materials Science: Materials In Medicine, Vol. 12, No. 1, 2001, pp. 7-13. doi:10.1023/A:1026792615665 